\title{
Study of Serum IP-10 Level as a Predictor for Non Alcoholic Steatohepatitis (NASH) In Diabetic and Non Diabetic Patients
}

\author{
Authors \\ El Said Ibrahim ${ }^{1}$, Doaa Hashad ${ }^{2}$, Perihan Salem ${ }^{1}$, Youssra Abo Elfotoh ${ }^{1}$ \\ ${ }^{1}$ Hepatobiliary Unite, Internal Medicine Department, Alexandria University \\ ${ }^{2}$ Clinical and Chemical Pathology Department, Alexandria University
}

\begin{abstract}
Non alcoholic liver disease (NAFLD) is a public health problem with high prevalence, high level of disability associated and high cost for the health system. Non alcoholic steato hepatitis (NASH) is among its spectrum where histopathological changes similar to those in alcoholic hepatitis were observed. Interferon gammainduced protein 10 (IP-10) is secreted in response to proinflammatory cytokines where it plays an important role in directing migration of cells, thus, function to regulate cell trafficking. This work aimed to study the significance of serum IP-10 level as a marker for NASH in diabetic and non-diabetic patients. Also, to correlate its level in the studied groups with clinical and laboratory findings.
\end{abstract}

Key words: NAFLD, NASH, cytokines, chemokines, IP-10, type II DM.

\section{Introduction}

Non alcoholic fatty liver disease (NAFLD) is one of the most common causes of chronic liver injury, ${ }^{(1)}$ with accumulation of triglycerides in the form of macro and micro vesicles, in more than $5 \%$ of the hepatocytes. ${ }^{(2)}$

NAFLD is more prevalent in patients with preexisting metabolic conditions as obesity, hypertriglyceridaemia, insulin resistance (IR) and type II diabetes mellitus (DM) where up to $69 \%$ of diabetic patients present with ultrasonographic NAFLD. ${ }^{(3)}$ Thus, it has become common to state that NAFLD is the "hepatic component" of metabolic syndrome (MS). ${ }^{(4)}$

NAFLD spectrum includes simple steatosis, nonalcoholic steato hepatitis (NASH), fibrosis, cirrhosis and hepatocellular carcinoma (HCC), ${ }^{(5)}$ where $10-25 \%$ of NAFLD patients develop NASH which carries an increased risk of $\mathrm{HCC},{ }^{6)}$ as
NASH was found to account for at least $13 \%$ of overall cases of HCC. ${ }^{(7)}$

Two types of NASH exist; primary NASH (which is associated with MS related conditions (as obesity, type II DM, and hypertriglyceridaemia) and secondary NASH (which occurs after obesity related intestinal surgery, rapid weight loss, total parenteral nutrition, lipodystrophy, willson's disease and drugs as amiodarone and thallium compounds. ${ }^{(8)}$

Theories for the pathogenesis of NASH were based on the '2-hit hypothesis'. The 'first hit', hepatic triglyceride accumulation, or steatosis, increases the susceptibility of liver injury mediated by 'second hits', such as inflammatory cytokines/adipokines, mitochondrial dysfunction and oxidative stress, which in turn lead to steatohepatitis and fibrosis. ${ }^{(9)}$ 
The role of hepatocyte cytokines in the progression of steatosis to NASH is supported by studies demonstrating that cytokines can induce all of the features associated with NASH, including neutrophil chemotaxis, hepatocyte apoptosis/necrosis, and stellate cell activation. ${ }^{(9)}$ Although there was no quantitative change in infiltrating cell types (as neutrophils, NK cells, NKT cells, and $\mathrm{T}$ cells), there was a significant influx of blood monocytes into the liver during NASH development. Kupffer cells and infiltrated monocytes participate as the first innate cells responding to hepatocyte injury due to lipid deposition. They are suggested to enhance hepatic lipid accumulation and liver injury through local secretion of IL- $1 \alpha$ and TNF $\alpha .{ }^{(10)}$ TNF- $\alpha$ enhances steatosis by inducing fatty acid uptake and reducing lipid export, leading to macro steatosis and liver damage. ${ }^{(11)}$

There is increasing evidence that indicates the existence of a chemokine (CXC) network in the liver which is involved in both physiological responses and, under certain circumstances, pathological and repair processes following hepatic injury. ${ }^{(12)}$

Chemokines are secreted in response to signals such as proinflammatory cytokines, where they play an important role in recruiting monocytes, neutrophils and lymphocytes which once stimulated followed by release of chemokines that directed migration of cells, expression of the appropriate chemokine receptors along a chemical liganed gradient known as chemokines gradient, and this allows cells to move toward high local concentration of chemokines. (13) Thus, chemokines play an important function to regulate cell trafficking. ${ }^{(14)}$

One particularly important pro-inflammatory chemokine is the CXC motif chemokine legend 10 (CXCL10) or interferon gamma-induced protein 10 (IP-10), which recruits inflammatory cells to the site of tissue damage. $(15,16)$ IP-10 has been implicated in the pathogenesis of hepatitis $\mathrm{C}$ virus (HCV) infection through interactions with the tolllike receptor (TLR) $2,{ }^{(17)}$ and in hepatitis B virus
(HBV) infection through the nuclear factor- $\kappa \mathrm{B}$ (NF- $\kappa \mathrm{B})$ pathway. ${ }^{(18)}$ In different types of liver injury, IP-10 is secreted by hepatocytes in areas of lobular inflammation, ${ }^{(19)}$ where neutralization of IP-10 accelerates liver regeneration. ${ }^{(20)}$

IP-10 is a small secretory protein of $8.7 \mathrm{KDa}(77$ amino acids) in its mature form that in humans is encoded by the IP-10 gene which is located on human chromosome 4 . IP-10 is secreted by several cell types, other than hepatocytes, in response to IFN- $\gamma$ and TNF $\alpha$; these cell types include monocytes, endothelial cells and fibroblasts. ${ }^{(21)}$

IP-10 elicits its effects by binding to the cell surface chemokine receptor CXCR3. There are two variants of CXCR3: CXCR3-A binds to the CXC chemokines CXCL9 (MIG), CXCL10 (IP10), and CXCL11 (I-TAC). Whereas, CXCR3-B can also bind to CXCL4 in addition to CXCL9, CXCL10, and CXCL11. CXCR3 are expressed primarily on activated $\mathrm{T}$ lymphocytes and NK cells, some epithelial cells and some endothelial cells. ${ }^{(2)}$

In a recent study, circulating levels of two chemokines (MCP1, IP-10) and two cytokines (IL6, TNF $\alpha$ ) are strongly up regulated in the liver tissue of NASH patients. ${ }^{(23)}$ Thus, TNF $\alpha$ silencing reduced the incidence of NASH development through the inhibition of IP-10 and MCP-1 chemokine production. ${ }^{(24)}$ In vitro neutralization of IP-10 using anti-CXCL10 mAb caused a dose dependent decrease in triglyceride secretion, ALT release and suppression of cellular oxidative stress with significant improvements in the prevention and regression of NASH. Thus, IP-10 is a potential target for the prevention and treatment of NASH. ${ }^{(25)}$

IP-10 has been demonstrated to be a key player in the pathogenesis of experimental NASH, (26) which correlates positively with obesity, IR, type I and type II DM. ${ }^{(27)}$ Thus, serum IP-10 level showed an evident increase in diabetic NAFLD/NASH patients. $(28,29)$ 


\section{Aim of work}

This work aimed to study the significance of serum interferon-gamma-inducible protein-10 (IP10) level as a marker for NASH in diabetic and non-diabetic patients. Also, to correlate its level in the studied groups with clinical and laboratory findings.

\section{Subjects and methods}

The study included 30 NASH diabetic patients (Group I), 30 NASH non-diabetic patients (Group II) presented to Hepatobiliary outpatient clinic, Alexandria Main University Hospital. Also, 30 age and sex matched healthy subjects with no evidence of DM or liver diseases were included in the study as a control group (Group III).

All patients were subjected to:

- Full history taking and clinical assessment stressing on exclusion criteria, together with calculation of the BMI using the following equation: $\mathrm{BMI}=$ Weight $(\mathrm{kg}) /$ Height $\left(\mathrm{m}^{2}\right)$.

- Biochemical studies including: fasting and 2 hours postprandial blood glucose level, liver profile (ALT; AST; alkaline phosphatase; total bilirubin; prothrombin activity and Serum albumin), and serum IP-10 level. ${ }^{(26)}$

- Abdominal ultrasound (US) to assess hepatic steatosis which can be graded into mild, moderate and severe.

\section{Statistical analysis of the data}

Data were fed to the computer and analyzed using IBMSPSS software package version 20.0. Qualitative data were described using number and percent. Quantitative data were described using range (minimum and maximum), mean, standard deviation and median. Significance of the obtained results was judged at the 5\% level. The used tests were: Chi-square test for categorical variables; to compare between different groups, Ftest (ANOVA) for normally quantitative variables; to compare between more than two studied groups, and Post Hoc test (LSD) for pairwise comparisons. Also, Student t-test and Pearson coefficient test for normally quantitative variables; to compare between two studied groups.

\section{Results}

Demographic data:

Table (1) showed that the study included 20 female and 10 male diabetic NASH patients (Group I) with mean age of 45.40 \pm 7.44 years, while the non-diabetic NASH patients (Group II) included 15 female and 15 male with mean age of $40.23 \pm 6.22$ years and the control group (Group III) included 17 females and 13 males with mean age of $42.0 \pm 7.90$ years.

Regarding the BMI, it showed close median in diabetic NASH and non-diabetic NASH patients (35.75, 34.55 respectively) with no statistical significant difference between both groups $\left(\mathrm{p}_{1}=0.085\right)$. While, a statically significant difference was detected between diabetic and nondiabetic NASH patients in comparison to control subjects $\left(\mathrm{p}_{2}<0.001, \mathrm{p}_{3}=0.001\right)$.

Blood pressure measurements:

Table (2) showed that the systolic blood pressure mean measurements in diabetic NASH patients (Group I) were $130.83 \pm 9.11 \mathrm{mmHg}$, in nondiabetic NASH patients (Group II) were $127.17 \pm 11.94 \mathrm{mmHg}$ and in control subjects (Group III) measurements were 121.33 \pm 7.76 $\mathrm{mmHg}$. Moreover the diastolic blood pressure mean measurements were $85.0 \pm 5.09 \mathrm{mmHg}$, $83.50 \pm 7.89 \mathrm{mmHg}$ and $80.17 \pm 5.94 \mathrm{mmHg}$ in the three studied groups respectively. These measurements showed increased systolic and diastolic blood pressure in diabetic and nondiabetic NASH patients (Group I and II) in comparison to control subjects (Group III).

Fasting plasma glucose (FPG) and post prandial glucose (PPG):

Table (3) showed an expected increase in FPG and PPG levels in diabetic NASH patients (Group I) with mean values of $149.20 \pm 21.54 \mathrm{mg} / \mathrm{dl}$ and $194.20 \pm 30.92 \mathrm{mg} / \mathrm{dl}$ which are higher than the values of non-diabetic NASH patients (Group II) 
$(92.07 \pm 5.97 \mathrm{mg} / \mathrm{dl}, 119.67 \pm 30.20 \mathrm{mg} / \mathrm{dl})$ and the control subjects (Group III) $(92.33 \pm 6.14 \mathrm{mg} / \mathrm{dl}$, $127.43 \pm 7.30 \mathrm{mg} / \mathrm{dl})$.

A statistically significant difference was observed between diabetic NASH patients (Group I) in comparison to non-diabetic NASH patients and control subjects (Group II and III).

\section{Lipid profile:}

Table (4) showed that serum levels of cholesterol and triglycerides increased significantly in NASH patients (Group I and II) in comparison to the control subjects (Group III). Also, the increase in diabetic NASH patients (mean of 205.83 \pm 22.12 $\mathrm{mg} / \mathrm{dl}, 269.23 \pm 63.14 \mathrm{mg} / \mathrm{dl}$ ) was more than in non-diabetic NASH patients (mean of $195.10 \pm 22.08 \mathrm{mg} / \mathrm{dl}, 232.97 \pm 42.0 \mathrm{mg} / \mathrm{dl}$ ). Thus, a statistically significant difference was reported between different studied groups.

\section{Liver function tests:}

Table (5) showed comparison between the different studied groups according to liver function tests. Serum ALT, AST and ALP mean levels were significantly increased in a stepwise fashion in control subjects, non-diabetic NASH and diabetic NASH patients respectively. A statistically significant difference was reported between different studied groups ( $\mathrm{p}<0.001)$.

Regarding total serum bilirubin level, it showed a similar median of $0.9 \mathrm{mg} / \mathrm{dl}$ in diabetic and nondiabetic NASH patients in comparison to a lower median of $0.7 \mathrm{mg} / \mathrm{dl}$ in control subjects, with a statistically significant difference between NASH patients and control subjects.

Prothrombin activity showed a median of $89.0 \%$, $92.50 \%$, 95.0\% among diabetic, non-diabetic NASH patients and control subjects respectively with a statistically significant difference between diabetic and non-diabetic NASH patients $(\mathrm{p}=0.024)$ and diabetic NASH patients and control subjects $(\mathrm{P}<0.001)$, while no statistically difference was reported between non-diabetic NASH and control subjects $(p=0.107)$.
Moreover, serum albumin level showed a close median among different studied groups (3.50, $3.65,3.45 \mathrm{~g} / \mathrm{dl}$ ) in diabetic NASH, non-diabetic NASH and control groups respectively with no statistically differences between different studied groups.

\section{Serum IP-10 level:}

Table (6) showed serum IP-10 levels among different studied groups. Its level was the highest among diabetic NASH patients $(338.31 \pm 139.85$ $\mathrm{pg} / \mathrm{ml}$ ) in comparison to non-diabetic NASH patients and control subjects (134.16 \pm 34.69 , $73.40 \pm 18.13 \mathrm{pg} / \mathrm{dl}$ respectively). An evident statistically significant difference was observed among different studied groups regarding serum IP-10 level $(\mathrm{P}<0.001)$.

Relation between serum IP-10 level and the degree of fatty infiltration detected by ultrasound in NASH groups:

Table (7) showed an evident stepwise increase in the median of serum IP-10 level in diabetic NASH patients with mild, moderate and severe fatty infiltration (209.0, 215.84, $478.25 \mathrm{pg} / \mathrm{ml}$ respectively). Also, similar stepwise increase in its median was observed in non-diabetic NASH patients (102.56, 129.71, $153.19 \mathrm{pg} / \mathrm{ml}$ respectively). A statistically significant difference was reported among the different degrees of fatty infiltration in diabetic and nondiabetic NASH groups $(\mathrm{P}<0.001)$.

Correlation between serum IP-10 level and different studied parameters in each group:

Serum IP-10 level was positively correlated with the degrees of fatty infiltration, serum levels of ALT and AST in diabetic and non-diabetic NASH patients. Otherwise, its level showed no correlation with different studied parameters.

Table (8) 
Table (1) Comparison between the three studied groups according to demographic data

\begin{tabular}{|c|c|c|c|c|c|c|c|}
\hline & \multicolumn{2}{|c|}{$\begin{array}{l}\text { Diabetic } \\
(\mathbf{n}=\mathbf{3 0})\end{array}$} & \multicolumn{2}{|c|}{$\begin{array}{l}\text { Non Diabetic } \\
(\mathbf{n}=\mathbf{3 0})\end{array}$} & \multicolumn{2}{|c|}{$\begin{array}{l}\text { Control } \\
(\mathbf{n}=\mathbf{3 0})\end{array}$} & \multirow[t]{2}{*}{$\mathbf{P}$} \\
\hline & No. & $\%$ & No. & $\%$ & No. & $\%$ & \\
\hline \multicolumn{8}{|l|}{ Gender } \\
\hline Male & 10 & 33.3 & 15 & 50.0 & 13 & 43.3 & \multirow{2}{*}{0.421} \\
\hline Female & 20 & 66.7 & 15 & 50.0 & 17 & 56.7 & \\
\hline Age (years) & \multirow{2}{*}{\multicolumn{2}{|c|}{$34.0-60.0$}} & \multirow{2}{*}{\multicolumn{2}{|c|}{$28.0-51.0$}} & \multirow{2}{*}{\multicolumn{2}{|c|}{$27.0-57.0$}} & \\
\hline Min. - Max. & & & & & & & \\
\hline Mean \pm SD & \multicolumn{2}{|c|}{$45.40 \pm 7.44$} & \multicolumn{2}{|c|}{$40.23 \pm 6.22$} & \multicolumn{2}{|c|}{$42.0 \pm 7.90$} & $0.014^{*}$ \\
\hline Median & \multicolumn{2}{|c|}{47.0} & \multicolumn{2}{|c|}{39.50} & \multicolumn{2}{|l|}{45.0} & \\
\hline Sig.bet.Grps & \multicolumn{6}{|c|}{$\mathrm{p}_{1}=0.004^{*}, \mathrm{p}_{2}=0.049^{*}, \mathrm{p}_{3}=0.346$} & \\
\hline \multicolumn{8}{|l|}{ BMI } \\
\hline Min. - Max. & \multicolumn{2}{|c|}{$29.80-41.0$} & \multicolumn{2}{|c|}{$27.0-41.0$} & \multicolumn{2}{|c|}{$23.70-37.80$} & \\
\hline Mean \pm SD & \multicolumn{2}{|c|}{$35.51 \pm 3.31$} & \multicolumn{2}{|c|}{$33.94 \pm 3.57$} & \multicolumn{2}{|c|}{$30.77 \pm 3.57$} & $<0.001^{*}$ \\
\hline Median & \multicolumn{2}{|l|}{35.75} & \multicolumn{2}{|c|}{34.55} & \multicolumn{2}{|c|}{30.30} & \\
\hline Sig.bet.Grps & \multicolumn{6}{|c|}{$\mathrm{p}_{1}=0.085, \mathrm{p}_{2}<0.001^{*}, \mathrm{p}_{3}=0.001^{*}$} & \\
\hline
\end{tabular}

$\chi^{2}:$ Chi square test

$\mathrm{p}_{1}$ : $\mathrm{p}$ value for comparing between diabetic and non diabetic

$\mathrm{p}_{2}: \mathrm{p}$ value for comparing between diabetic and control

$\mathrm{p}_{3}$ : $\mathrm{p}$ value for comparing between non diabetic and control

*: Statistically significant at $\mathrm{p} \leq 0.05$

Table (2) Comparison between the three studied groups according to blood pressure measurements

\begin{tabular}{|c|c|c|c|c|}
\hline & $\begin{array}{l}\text { Diabetic } \\
(\mathbf{n}=\mathbf{3 0})\end{array}$ & $\begin{array}{l}\text { Non Diabetic } \\
(\mathbf{n}=\mathbf{3 0})\end{array}$ & $\begin{array}{l}\text { Control } \\
(\mathbf{n}=\mathbf{3 0})\end{array}$ & $\mathbf{P}$ \\
\hline \multicolumn{5}{|l|}{ Systolic (mmHg) } \\
\hline Min. - Max. & $120.0-150.0$ & $100.0-150.0$ & $110.0-140.0$ & \\
\hline Mean \pm SD & $130.83 \pm 9.11$ & $127.17 \pm 11.94$ & $121.33 \pm 7.76$ & $0.001^{*}$ \\
\hline Median & 130.0 & 122.50 & 120 & \\
\hline Sig.bet. Grps & \multicolumn{3}{|c|}{$\mathrm{p}_{1}=0.149, \mathrm{p}_{2}<0.001^{*}, \mathrm{p}_{3}=0.023^{*}$} & \\
\hline \multicolumn{5}{|l|}{ Diastolic (mmHg) } \\
\hline Min. - Max. & $80.0-95.0$ & $70-100.0$ & $70-90$ & \\
\hline Mean \pm SD & $85.0 \pm 5.09$ & $83.50 \pm 7.89$ & $80.17 \pm 5.94$ & $0.014^{*}$ \\
\hline Median & 85.0 & 80.0 & 80 & \\
\hline Sig.bet. Grps & \multicolumn{3}{|c|}{$\mathrm{p}_{1}=0.368, \mathrm{p}_{2}=0.004^{*}, \mathrm{p}_{3}=0.047^{*}$} & \\
\hline
\end{tabular}

$\mathrm{p}_{1}: \mathrm{p}$ value for comparing between diabetic and non diabetic

$\mathrm{p}_{2}$ : $\mathrm{p}$ value for comparing between diabetic and control

$\mathrm{p}_{3}: \mathrm{p}$ value for comparing between non diabetic and control

*: Statistically significant at $\mathrm{p} \leq 0.05$ 
Table (3): Comparison between the three studied groups according to FPG and PPG levels

\begin{tabular}{|c|l|l|l|l||}
\hline & $\begin{array}{l}\text { Diabetic } \\
(\mathbf{n}=\mathbf{3 0})\end{array}$ & $\begin{array}{l}\text { Non Diabetic } \\
(\mathbf{n}=\mathbf{3 0})\end{array}$ & $\begin{array}{l}\text { Control } \\
(\mathbf{n}=\mathbf{3 0})\end{array}$ & $\mathbf{P}$ \\
\hline FPG (mg/dl) & \multicolumn{3}{|l||}{} & \\
Min. - Max. & $110.0-200.0$ & $79.0-100.0$ & $79.0-100.0$ \\
Mean \pm SD. & $149.20 \pm 21.54$ & $92.07 \pm 5.97$ & $92.33 \pm 6.14$ & $<0.001^{*}$ \\
Median & 150.50 & 91.50 & 92.0 & \\
\hline Sig.bet. Grps & $\mathrm{p}_{1}<0.001^{*}, \mathrm{p}_{2}<0.001^{*}, \mathrm{p}_{3}=0.939$ & \\
\hline PPG (mg/dl) & \multicolumn{3}{|l}{} & \\
Min. - Max. & $120.0-270.0$ & $11.0-140.0$ & $110.0-140.0$ & $<0.001^{*}$ \\
Mean \pm SD. & $194.20 \pm 30.92$ & $119.67 \pm 30.20$ & $127.43 \pm 7.30$ & \\
Median & 190.0 & 129.0 & 129.0 & \\
\hline Sig.bet.Grps & $\mathrm{p}_{1}<0.001^{*}, \mathrm{p}_{2}<0.001^{*}, \mathrm{p}_{3}=0.238$ & \\
\hline
\end{tabular}

$\mathrm{p}_{1}: \mathrm{p}$ value for comparing between diabetic and non diabetic

$\mathrm{p}_{2}: \mathrm{p}$ value for comparing between diabetic and control

$\mathrm{p}_{3}$ : $\mathrm{p}$ value for comparing between non diabetic and control

*: Statistically significant at $\mathrm{p} \leq 0.05$

Table (4): Comparison between the three studied groups according to lipid profile

\begin{tabular}{|c|c|c|c|c|}
\hline & $\begin{array}{r}\text { Diabetic } \\
(\mathrm{n}=\mathbf{3 0})\end{array}$ & $\begin{array}{r}\text { Non Diabetic } \\
(\mathbf{n}=\mathbf{3 0})\end{array}$ & $\begin{array}{l}\text { Control } \\
(\mathbf{n}=30)\end{array}$ & $\mathbf{p}$ \\
\hline Cholesterol (mg/dl) & & & & \\
\hline Min. - Max. & $175.0-250.0$ & $150.0-240.0$ & $129.0-200.0$ & \\
\hline Mean \pm SD & $205.83 \pm 22.12$ & $195.10 \pm 22.08$ & $151.83 \pm 16.08$ & $<0.001^{*}$ \\
\hline Median & 203.0 & 191.50 & 148.50 & \\
\hline Sig.bet. Grps & \multicolumn{3}{|c|}{$\mathrm{p}_{1}=0.043^{*}, \mathrm{p}_{2}=0.001^{*}, \mathrm{p}_{3}<0.001^{*}$} & \\
\hline \multicolumn{5}{|l|}{$\begin{array}{r}\text { Triglycerides } \\
\text { (mg/dl) }\end{array}$} \\
\hline Min. - Max. & $158.0-416.0$ & $150.0-289.0$ & $79.0-189.0$ & \\
\hline Mean \pm SD & $269.23 \pm 63.14$ & $232.97 \pm 42.0$ & $130.70 \pm 28.97$ & $<0.001^{*}$ \\
\hline Median & 267.50 & 247.50 & 135.0 & \\
\hline Sig.bet.Grps & \multicolumn{3}{|c|}{$\mathrm{p}_{1}=0.004^{*}, \mathrm{p}_{2}<0.001^{*}, \mathrm{p}_{3}<0.001^{*}$} & \\
\hline
\end{tabular}

$\mathrm{p}_{1}: \mathrm{p}$ value for comparing between diabetic and non diabetic

$\mathrm{p}_{2}$ : $\mathrm{p}$ value for comparing between diabetic and control

$\mathrm{p}_{3}$ : $\mathrm{p}$ value for comparing between non diabetic and control

*: Statistically significant at $\mathrm{p} \leq 0.05$ 
Table (5) Comparison between the three studied groups according to liver function tests

\begin{tabular}{|c|c|c|c|c|}
\hline & $\begin{array}{l}\text { Diabetic } \\
(\mathbf{n}=30)\end{array}$ & $\begin{array}{l}\text { Non Diabetic } \\
(\mathbf{n}=30)\end{array}$ & $\begin{array}{l}\text { Control } \\
(\mathbf{n}=30)\end{array}$ & $\mathbf{p}$ \\
\hline $\operatorname{ALT}(\mathbf{U} / \mathbf{L})$ & & & & \\
\hline Min. - Max. & $85.0-140.0$ & $65.0-118.0$ & $17.0-40.0$ & \\
\hline Mean \pm SD & $103.50 \pm 12.97$ & $87.63 \pm 12.01$ & $29.83 \pm 6.28$ & $<0.001^{*}$ \\
\hline Median & 100.0 & 88.50 & 28.0 & \\
\hline Sig.bet. Grps & \multicolumn{3}{|c|}{$\mathrm{P}_{1}<0.001^{*}, \mathrm{p}_{2}<0.001^{*}, \mathrm{p}_{3}<0.001^{*}$} & \\
\hline \multicolumn{5}{|l|}{ AST (U/L) } \\
\hline Min. - Max. & $45.0-75.0$ & $30.0-70.0$ & $0.70-30.0$ & \\
\hline Mean \pm SD & $63.13 \pm 8.13$ & $53.67 \pm 10.76$ & $20.40 \pm 8.34$ & $<0.001^{*}$ \\
\hline Median & 65.0 & 55.50 & 24.0 & \\
\hline Sig.bet. Grps & \multicolumn{3}{|c|}{$\mathrm{P}_{1}<0.001^{*}, \mathrm{p}_{2}<0.001^{*}, \mathrm{p}_{3}<0.001^{*}$} & \\
\hline \multicolumn{5}{|l|}{$\operatorname{ALP}(I U / L)$} \\
\hline Min. - Max. & $69.0-200.0$ & $78.0-186.0$ & $40.0-147.0$ & \\
\hline Mean \pm SD & $131.20 \pm 31.63$ & $111.33 \pm 27.49$ & $89.50 \pm 28.0$ & $<0.001^{*}$ \\
\hline Median & 130.0 & 100.50 & 86.50 & \\
\hline Sig.bet. Grps & \multicolumn{3}{|c|}{$\mathrm{p}_{1}=0.010^{*}, \mathrm{p}_{2}<0.001^{*}, \mathrm{p}_{3}=0.005^{*}$} & \\
\hline \multicolumn{5}{|l|}{$\begin{array}{ll}\text { Total } & \text { billirubin } \\
(\mathrm{mg} / \mathrm{dl}) & \end{array}$} \\
\hline Min. - Max. & $0.20-1.5$ & $0.40-1.20$ & $0.20-1.10$ & \\
\hline Mean \pm SD & $1.06 \pm 1.33$ & $0.89 \pm 0.21$ & $0.73 \pm 0.21$ & $\begin{array}{l}{ }^{\mathrm{KW}} \mathrm{p} \\
0.011^{*}\end{array}$ \\
\hline Median & 0.90 & 0.90 & 0.70 & \\
\hline Sig.bet. Grps & \multicolumn{3}{|c|}{$\mathrm{p}_{1}=0.515, \mathrm{p}_{2}=0.0 .31^{*}, \mathrm{p}_{3}<0.004^{*}$} & \\
\hline \multicolumn{5}{|l|}{ PT activity (\%) } \\
\hline Min. - Max. & $75.0-97.0$ & $67.0-98.0$ & $79.0-100.0$ & \multirow{3}{*}{$0.001^{*}$} \\
\hline Mean \pm SD & $87.73 \pm 6.97$ & $91.40 \pm 6.40$ & $94.0 \pm 5.02$ & \\
\hline Median & 89.0 & 92.50 & 95.0 & \\
\hline Sig.bet. Grps & \multicolumn{3}{|c|}{$\mathrm{p}_{1}=0.024^{*}, \mathrm{p}_{2}<0.001^{*}, \mathrm{p}_{3}=0.107$} & \\
\hline \multicolumn{5}{|l|}{ ALB (g/dl) } \\
\hline Min. - Max. & $2.60-4.10$ & $2.80-4.10$ & $2.40-4.0$ & \\
\hline Mean \pm SD & $3.39 \pm 0.45$ & $3.56 \pm 0.41$ & $3.41 \pm 0.42$ & 0.265 \\
\hline Median & 3.50 & 3.65 & 3.45 & \\
\hline
\end{tabular}

KW: Kruskal Wallis test, Sig. bet. grps was done using Mann Whitney test $\mathrm{p}_{1}$ : $\mathrm{p}$ value for comparing between diabetic and non diabetic $\mathrm{p}_{2}$ : $\mathrm{p}$ value for comparing between diabetic and control $\mathrm{p}_{3}$ : $\mathrm{p}$ value for comparing between non diabetic and control *: Statistically significant at $\mathrm{p} \leq 0.05$ 
Table (6) Comparison between the three studied groups according to serum IP-10 level

\begin{tabular}{|c|l|l|l|l|}
\hline & $\begin{array}{l}\text { Diabetic } \\
(\mathbf{n = 3 0})\end{array}$ & $\begin{array}{l}\text { Non-diabetic } \\
(\mathbf{n = 3 0})\end{array}$ & $\begin{array}{l}\text { Control } \\
(\mathbf{n}=\mathbf{3 0})\end{array}$ & $\mathbf{p}$ \\
\hline IP-10 $(\mathbf{p g} / \mathbf{m l})$ & & & & \\
Min. - Max. & $143.07-489.70$ & $93.12-254.25$ & $43.15-106.11$ & \\
Mean \pm SD. & $338.31 \pm 139.85$ & $134.16 \pm 34.69$ & $73.40 \pm 18.13$ & $<0.001^{*}$ \\
Median & 360.15 & 128.87 & 73.99 & \\
\hline Sig.bet. Grps & $\mathrm{p}_{1}<0.001^{*}, \mathrm{p}_{2}<0.001^{*}, \mathrm{p}_{3}=0.006^{*}$ & & \\
\hline
\end{tabular}

$\mathrm{p}_{1}: \mathrm{p}$ value for comparing between diabetic and non diabetic

$\mathrm{p}_{2}$ : $\mathrm{p}$ value for comparing between diabetic and control

$p_{3}$ : $p$ value for comparing between non diabetic and control

*: Statistically significant at $\mathrm{p} \leq 0.05$

Table (7) Relation between serum IP-10 level and the degree of fatty infiltration detected by ultrasound in NASH groups

\begin{tabular}{|c|c|c|c|c|}
\hline \multirow{2}{*}{ IP10 } & \multicolumn{3}{|c|}{ Degree of fatty infiltration } & \\
\hline & Mild & Moderate & Severe & \\
\hline Diabetic & $(n=4)$ & $(\mathbf{n}=8)$ & $(n=18)$ & \\
\hline Min. - Max. & $160.12-305.10$ & $143.07-429.36$ & $156.82-489.70$ & \\
\hline Mean \pm SD & $220.80 \pm 70.85$ & $230.36 \pm 88.76$ & $412.41 \pm 121.20$ & $<0.001^{*}$ \\
\hline Median & 209.0 & 215.84 & 478.25 & \\
\hline Non-diabetic & $(\mathbf{n}=\mathbf{8})$ & $(n=13)$ & $(\mathbf{n}=9)$ & \\
\hline Min. - Max. & $93.12-122.86$ & $108.55-133.91$ & $124.52-254.25$ & \\
\hline Mean \pm SD & $105.46 \pm 9.17$ & $126.84 \pm 7.12$ & $170.26 \pm 42.84$ & $<0.001^{*}$ \\
\hline Median & 102.56 & 129.71 & 153.19 & \\
\hline
\end{tabular}

*: Statistically significant at $\mathrm{p} \leq 0.05$

Table (8): Correlation between serum IP-10 level and different studied parameters in each group

\begin{tabular}{||l|l|l|l|l||}
\hline \multirow{2}{*}{} & & \multicolumn{2}{l|}{ IP10 } \\
\cline { 3 - 5 } \multicolumn{2}{|l|}{ Age } & Diabetic & Non Diabetic & Control \\
\hline \multirow{2}{*}{ FBG } & $\mathbf{r}$ & 0.148 & 0.111 & -0.146 \\
& $\mathbf{p}$ & 0.435 & 0.558 & 0.441 \\
\hline \multirow{2}{*}{ PPG } & $\mathbf{r}$ & 0.196 & 0.211 & -0.015 \\
& $\mathbf{p}$ & 0.300 & 0.263 & 0.937 \\
\hline ALT & $\mathbf{r}$ & 0.122 & 0.006 & -0.057 \\
& $\mathbf{p}$ & 0.519 & 0.974 & 0.766 \\
\hline
\end{tabular}

El Said Ibrahim et al JMSCR Volume 04 Issue 02 February 


\begin{tabular}{||l|l|l|l|l||}
\hline \multirow{2}{*}{ AST } & $\mathbf{p}$ & $0.001^{*}$ & $0.002^{*}$ & $0.030^{*}$ \\
\hline \multirow{2}{*}{ Cholesterol } & $\mathbf{r}$ & $0.491^{*}$ & $0.593^{*}$ & 0.096 \\
& $\mathbf{p}$ & $0.006^{*}$ & $<0.001^{*}$ & 0.615 \\
\hline \multirow{2}{*}{ Triglycerides } & $\mathbf{r}$ & 0.151 & 0.094 & 0.299 \\
& $\mathbf{p}$ & 0.427 & 0.621 & 0.108 \\
\hline \multirow{2}{*}{ PT } & $\mathbf{r}$ & 0.183 & 0.082 & -0.056 \\
\hline \multirow{2}{*}{ BMI } & $\mathbf{p}$ & 0.332 & 0.665 & 0.768 \\
\hline \multirow{2}{*}{ Systolic BP } & $\mathbf{r}$ & -0.341 & -0.317 & 0.162 \\
\hline \multirow{2}{*}{ Diastolic BP } & $\mathbf{p}$ & 0.065 & 0.088 & 0.394 \\
\hline \multirow{2}{*}{ Degree of steatosis } & $\mathbf{r}$ & 0.215 & 0.172 & -0.033 \\
& $\mathbf{p}$ & 0.255 & 0.364 & 0.863 \\
\hline & $\mathbf{r}$ & 0.141 & 0.204 & -0.069 \\
\hline & $\mathbf{p}$ & 0.457 & 0.280 & 0.718 \\
\hline & $\mathbf{r}$ & 0.209 & 0.300 & 0.035 \\
\hline
\end{tabular}

r: Pearson coefficient

*: Statistically significant at $\mathrm{p} \leq 0.05$

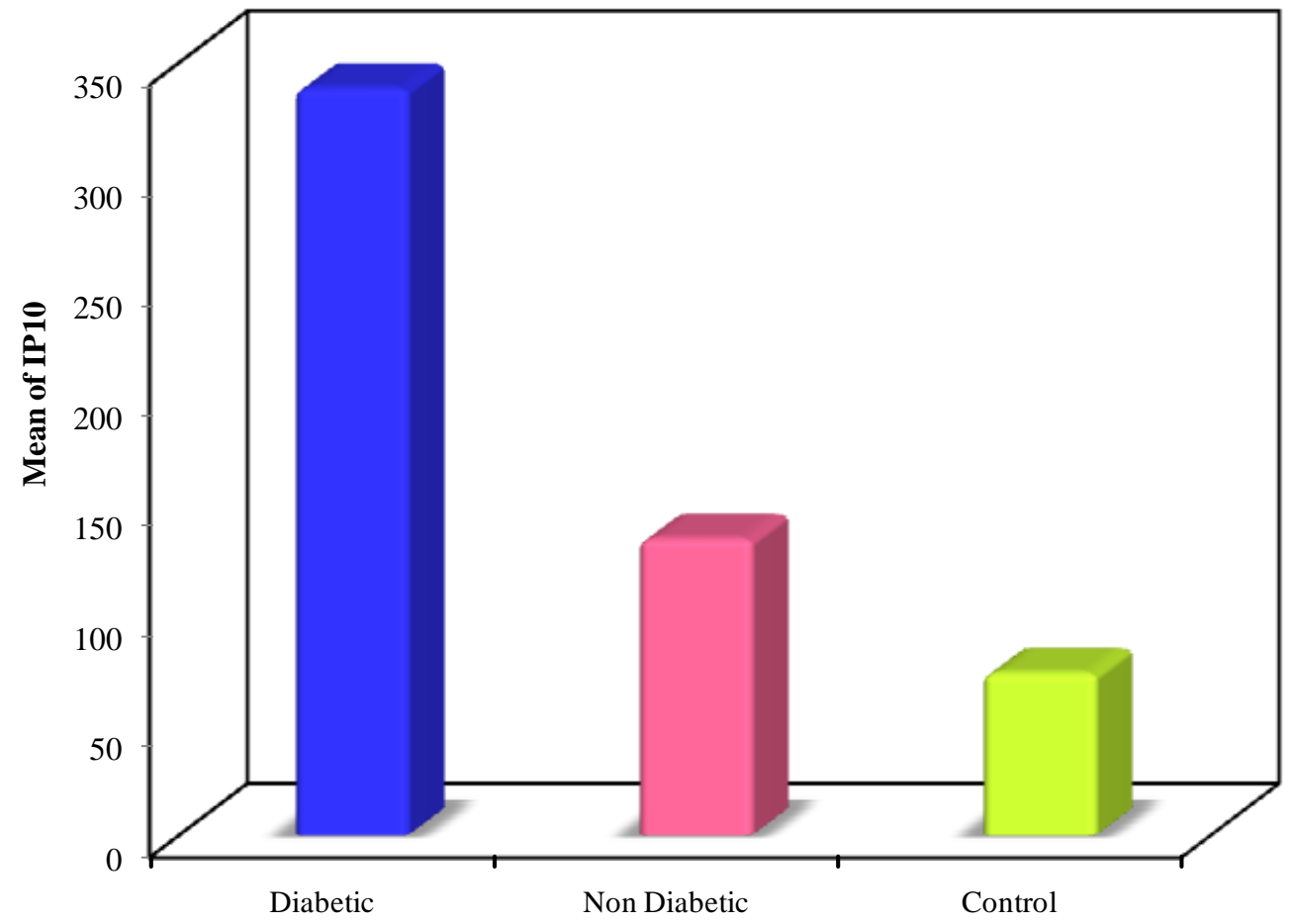

Figure (1): Comparison between the three studied groups according to serum IP-10 level 


\section{Discussion}

Non-alcoholic steatohepatitis (NASH) is a severe form of hepatic steatosis characterized by lipid accumulation and necroinflammation. (30) Inflammation may result from oxidative stress and pro-inflammatory cytokines and chemokines, which perpetuate liver injury and lead to fibrosis. (20)

One of the important pro-inflammatory cytokine associated with lipotoxicity is the interferon gamma induced protein (IP-10), which recruits inflammatory cells to the site of tissue damage. ${ }^{(15)}$ In various types of liver injury, IP-10 is secreted by hepatocytes and its neutralization accelerates liver regeneration. ${ }^{(31)}$

Regarding gender, the present study included 35 female and 25 male NASH patients. In agreement with our study, Sligte KT et al ${ }^{(32)}$ found that NASH occurs more frequently in females. On the other hand, Arun $\mathrm{J}$ et al ${ }^{(31)}$ reported that the prevalence of NASH in males and females was (60.3\% and $30.9 \%$ respectively).

The mean age of the diabetic and non-diabetic NASH patients in the present study was $45.40 \pm 7.44$ years and $40.23 \pm 6.22$ years respectively. While, in a study by Bazick $\mathrm{J}$ et al, (33) the average age of NASH patients with diabetes was much higher $(52.4 \pm 10.3$ years $)$.

In the present study, BMI showed close median in diabetic and non-diabetic NASH patients (35.75, 34.55 respectively), with no statistical significant difference between both groups. However, a statistical significant difference was reported between diabetic and non-diabetic NASH patients in comparison to control subjects. In accordance with our results, Luyckx FH et al ${ }^{(34)}$ reported that NASH is frequently associated with obesity (especially visceral fat), type II DM and is intimately related to markers of the IR syndrome.

In this work, the measurements of systolic and diastolic blood pressure were increased in diabetic and non-diabetic NASH patients in comparison to control subjects. In agreement with our results, Marchinsi G et al ${ }^{(35)}$ and Hsien-Liang $\mathrm{H}$ et al (36) reported a positive correlation between NASH and high blood pressure.

In our study, diabetic NASH patients showed an expected increase in FBG and PPG levels in comparison to non-diabetic NASH and control groups. Many studies demonstrated the link between NASH and type II DM, Gupte A et al ${ }^{(37)}$ and Kenneth $\mathrm{C}^{(38)}$ reported that the prevalence of $\mathrm{NASH}$ is high among type II DM patients where NASH is a frequently overlooked complication of type II DM. Also, Prashanth $\mathrm{M}$ et al (39) and Younossi $\mathrm{ZM}^{(40)}$ found that the prevalence of NAFLD, NASH and fibrosis in their cohort of type II DM patients is high. It was evident from their study that NASH and advanced fibrosis can occur in diabetic patients without any symptoms, signs or laboratory abnormalities in their liver functions. Since advanced fibrosis is unlikely to regress spontaneously, these patients have the risk of progression to cirrhosis, HCC and liver cell failure. Again, Park SK, ${ }^{(41)}$ Jason MH et al ${ }^{(42)}$ and Ong JP et al ${ }^{(43)}$ proved that NASH is an independent risk factor for the development of type II DM.

Regarding serum levels of cholesterol and triglyceride in the present study, they were significantly high in NASH patients in comparison to the control group. Also, the increase in diabetic NASH patients was more than in non-diabetic NASH patients with a statistical significant difference between different studied groups. In agreement with our findings, Kotronen A et al (44) reported similar findings of a stepwise increase in the lipid profile from the control group to the nondiabetic NASH patients till the diabetic NASH patients.

In the present study, serum ALT; AST and ALP showed a significant increase in a stepwise fashion in control; non-diabetic NASH and diabetic NASH groups respectively with a statistical significant difference between different studied groups. While, total serum bilirubin level, prothrombin activity and serum albumin level in NASH patients were within the normal ranges. 
In agreement with our study, Harris $\mathrm{EH}^{(45)}$ found that type II DM patients had a higher incidence of liver function tests abnormalities than nondiabetic individuals. However, Siddharth V et al ${ }^{(46)}$ found that no ideal ALT cut off that would best predict underlying liver disease severity. Moreover, Prashanth $\mathrm{M}$ et al ${ }^{(39)}$ reported that only ALT was shown to be significantly associated with NASH in patients with type II DM. Also, in accordance with our findings, Kocabay GL et al (47) found that higher level of ALP may be considered as a risk factor linked to hepatic fibrosis in patients with NASH and type II DM.

Regarding other studied liver function tests, Bazick J et al ${ }^{(33)}$ found that total bilirubin level and albumin levels in NASH patients were within the normal range.

In the present work, the mean of serum IP-10 level was the highest among the diabetic NASH patients $(338.31 \pm 139.85 \mathrm{pg} / \mathrm{ml})$ in comparison to non-diabetic NASH patients and control subjects $(134.16 \pm 34.69 \mathrm{pg} / \mathrm{ml}$ and $73.40 \pm 18.13 \mathrm{pg} / \mathrm{ml}$ respectively) with an evident statistical significant difference among different studied groups. Similar to our results, Chia-Chu $\mathrm{C}$ et al ${ }^{(26)}$ proved a stepwise increase in serum IP-10 level from control subjects to non-diabetic NAFLD and diabetic NAFLD patients.

In relation to diabetes, Sajadi SM et al, ${ }^{(48)}$ Jingfang L et al, ${ }^{(49)}$ and XU H et al ${ }^{(50)}$ showed that serum levels of IP-10 were elevated in type II DM patients (especially those with diabetic retinopathy and nephropathy) in comparison to non-diabetics.

Moreover, Morimoto $\mathrm{J}$ et al ${ }^{(27)}$ demonstrated that IP-10 neutralization enhanced $\beta$ cell proliferation and suppressed diabetes occurrence in non-obese diabetic mice. Also, Bertola A et al ${ }^{(23)}$ found that IP-10 gene was significantly up regulated in the liver of obese patients with NASH, and Zhang X et al ${ }^{(24)}$ found that IP-10 was an independent risk factor for the development of NASH.

In the present study, serum IP-10 level was positively correlated with the degrees of fatty infiltration, where its level showed a stepwise increase from mild to moderate and severe steatosis. Moreover, it is positively correlated with serum levels of ALT and AST in diabetic and non-diabetic NASH patients. Otherwise, its level showed no correlation with different studied parameters.

On the contrast to our results, Zhang $\mathrm{X}$ et al ${ }^{(24)}$ and Chia-Chu $\mathrm{C}$ et al ${ }^{(26)}$ reported a significant correlation between serum IP-10 level and a wide range of metabolic parameters as fasting serum insulin, HOMA-IR, TG.

\section{Conclusion}

- IP-10 is secreted by hepatocytes in areas of lobular inflammation, and its neutralization showed improvement in the prevention and progression of steatohepatitis together with acceleration of liver regeneration. This indicates the potential role of IP-10 in the development of intrahepatic inflammation.

- IP-10 is up regulated in NASH patients and correlates positively with the degree of fatty infiltration. This finding suggests that IP-10 could be a pivotal molecule that facilitates transition from benign steatosis to progressive hepatocellular damage and inflammation in NASH.

\section{Recommendations}

- Identification of the pro-inflammatory cytokines, which are associated with lipotoxicity, may improve our understanding of the pathogenesis of $\mathrm{NASH}$, enabling the development of novel pharmacological treatments.

- In vitro neutralization of IP-10 using antiCXCL10 mAb caused a dose dependent decrease in triglyceride secretion, ALT release and suppression of cellular oxidative stress with significant improvements in the prevention and regression of NASH. Thus, IP-10 is a potential target for the prevention and treatment of NASH. However, further studies are needed in this respect. 


\section{References}

1. Okanoue T, Umemura A, Yasui K, Itoh Y. Nonalcoholic fatty liver disease and nonalcoholic steatohepatitis in Japan. J Gastroen Hepatol 2011; 26: 153-62.

2. Brunt EM. Nonalcoholic fatty liver disease: what the pathologist can tell the clinician. Dig Dis 2012; 30(1): 61-8.

3. Leite NC, Salles GF, Araujo AL, VillelaNogueira CA, Cardoso CR. Prevalence and associated factors of non-alcoholic fatty liver disease in patients with type-2 diabetes mellitus. Liver Int 2009; 29: 1139.

4. Otgonsuren M, Stepanova M, Gerber L, Younossi ZM. Anthropometric and clinical factors associated with mortality in subjects with nonalcoholic fatty liver disease. Dig Dis Sci 2013; 58: 1132-40.

5. Yilmaz Y. Review article: is non-alcoholic fatty liver disease a spectrum, or are steatosis and non-alcoholic steatohepatitis distinct conditions? Aliment Pharmacol Ther 2012; 36: 815-23.

6. Ekstedt M, Franzen LE, Mathiesen UL, Thorelius L, Holmqvist M, Bodemar G. Long-term follow-up of patients with NAFLD and elevated liver enzymes. Hepatology 2006; 44: 865-73.

7. Marrero JA, Fontana RJ, $\mathrm{Su}$ GL, Conjeevaram HS, Emick DM, Lok AS. NAFLD may be a common underlying liver disease in patients with hepatocellular carcinoma in the United States. Hepatology 2002; 36: 1349-54.

8. Chitturi S, Farrell GC. Etiopathogenesis of non alcoholic steatohepatitis. Semin Liver Dis 2001; 21(1): 27-41.

9. Day CP. From fat to inflammation. Gastroenterology 2006; 130: 207-10.

10. Tosello TAC, Landes SG, Nguyen V, Novobrantseva T, Young S. Kuppfer Cells Trigger Nonalcoholic Steatohepatitis Development in Diet-induced Mouse Model through Tumor Necrosis Factor- $\alpha$
Production. J Biol Chem 2012; 287(48): 40161-72

11. Chen X, Xun K, Chen L, Wang Y. TNFalpha, a potent lipid metabolism regulator. Cell Biochem Funct 2009; 27(7): 407-16.

12. Hassanshahi G, Jafarzadeh A, Ghorashi Z, Zia Sheikholeslami N, Dickson AJ. Expression of IP-10 chemokine is regulated by pro-inflammatory cytokines in cultured hepatocytes. Iran J Allergy Asthma Immunol 2007; 6(3): 115-21.

13. Callewaere C, Banisadr G, Rostene W, Parsadaniantz SM. Chemokines and chemokine receptors in the brain: implication in neuroendocrine regulation. $\mathbf{J}$ Mol Endocrinol 2007; 38: 355-63.

14. Deshmane SL, Kremlev S, Amini S, Sawaya BE. Monocyte chemotractant protein-1 (MCP-1): An overview. Journal of Interferon \& Cytokine Research 2008; 29(6): 313-26.

15. Neville LF, Mathiak G, Bagasra O. The immunobiology of interferon-gamma inducible protein $10 \mathrm{kD}$ (IP-10): a novel, pleiotropic member of the C-X-C chemokine superfamily. Cytokine Growth Factor Rev 1997; 8: 207-19.

16. Luster AD, Unkeless JC, Ravetch JV. Gamma-interferon transcriptionally regulates an early response gene containing homology to platelet proteins. Nature 1985; 315: 672-6.

17. Abe T, Fukuhara T, Wen X, Ninomiya A, Moriishi K, Maehara Y, et al. CD44 participates in IP-10 induction in cells in which hepatitis $\mathrm{C}$ virus RNA is replicating, through an interaction with Toll-like receptor 2 and hyaluronan. J Virol 2010; $86: 6159-70$.

18. Zhou Y, Wang S, Ma JM, Lei Z, Zhu HF, Lei $\mathrm{P}$, et al. Hepatitis $\mathrm{B}$ virus protein $\mathrm{X}$ induced expression of the CXC chemokine IP-10 is mediated through activation of NF-kappa B and increases migration of 
leukocytes. J Biol Chem 2010; 285: 12159-68.

19. Harvey CE, Post JJ, Palladinetti P, Freeman AJ, Ffrench RA, Kumar RK, et al. Expression of the chemokine IP-10 (CXCL10) by hepatocytes in chronic hepatitis $\mathrm{C}$ virus infection correlates with histological severity and lobular inflammation. J Leukoc Biol 2003; 74: 3609.

20. Yoneyama H, Kai Y, Koyama J, Suzuki K, Kawachi K, Narumi S, et al. Neutralization of CXCL10 accelerates liver regeneration in carbon tetrachloride-induced acute liver injury. Med Mol Morphol 2007; 40: 1917.

21. Nagpal ML, Davis J, Lin T. Overexpression of CXCL10 in human prostate $\mathrm{LNCaP}$ cells activates its receptor (CXCR3) expression and inhibits cell proliferation. Molecular basis of disease 2006; 1762: 811-8.

22. Booth V, Keizer DW, Kamphuis MB, Clark-Lewis I, Sykes BD. The CXCR3 binding chemokine IP-10/CXCL10: structure and receptor interactions. Biochemistry 2002; 41(33): 10418-25.

23. Bertola A, Bonnafous S, Anty R, Patouraux S, Saint-Paul MC, Iannelli A, et al. Hepatic Expression Patterns of Inflammatory and Immune Response Genes Associated with Obesity and NASH in Morbidly Obese Patients. Plos 2010; 10: 1371.

24. Zhang X, Shen J, Man K, Chu ES, Yau TO, Sung JC, Go MY, Deng J, Lu L, Wong VW, Sung JJ, Farrell G, Yu J. CXCL10 plays a key role as an inflammatory mediator and a non-invasive biomarker of non-alcoholic steatohepatitis. J Hepatol 2014; 61: 1365-75.

25. Herder C, Haastert B, Müller-Scholze
S, Koenig
$\mathrm{W}$, Thorand
B, Holle

R, Wichmann HE, Scherbaum WA, Martin

$\mathrm{S}$, Kolb H. Association of systemic chemokine concentrations with impaired glucose tolerance and type 2 diabetes: results from the Cooperative Health Research in the Region of Augsburg Survey S4 (KORA S4). Diabetes 2005; 54(2): 11-7.

26. Chia-Chu C, Chia-Lin W, Wei-Wen S, Kai-Lun S, Der-Cherng T, Chen-Te C, Ting-Yu C, Chew-Teng K , Hung-Ming W. Interferon gamma-induced protein 10 is associated with insulin resistance and incident diabetes in patients with nonalcoholic fatty liver disease. Scientific Reports 2015; 5: 10096.

27. Morimoto J, Yoneyama H, Shimada A, ShigiharaT, Yamada S, Oikawa Y. CXC chemokine ligand 10 neutralization suppresses the occurrence of diabetes in nonobese diabetic mice through enhanced beta cell proliferation without affecting insulitis. J Immunol 2004; 173: 7017-24.

28. Shimada A, Morimoto J, Kodama K, Suzuki R, Oikawa Y, Funae O, Kasuga A. Elevated serum IP-10 levels observed in type 1 diabetes. Diabetes care 2001; 24: 510-5.

29. Pagano G, Pacini G, Musso G, GambinoP,Mecca F, Depetris N, Cassader M, David E, Cavallo-PerinP, Rizzetto M.D.Nonalcoholic steatohepatitis, insulin resistance, and metabolic syndrome: Furthe evidence for an etiologic association .Hepatology 2002; 2:367-72.

30. Hintermann E, Bayer M, Pfeilschifter JM, Luster AD, Christen U. CXCL10 promotes liver fibrosis by prevention of NK cell mediated hepatic stellate cell inactiveation. J Autoimmun 2010; 35: 424-35.

31. Arun J, Clements RH, Lazenby AJ, Leeth RR, Abrams GA. The prevalence of nonalcoholic steatohepatitis is greater in morbidly obese men compared to women. Obes Surg 2006; 16(10): 1351-8.

32. Sligte KT, Bourass I, Sels JP, Driessen A, Stockbrugger RW, Koek GH. Non- 
alcoholic steatohepatitis: review of a growing medical problem. Eur $\mathrm{J}$ Intern Med 2004; 15(1): 10-21.

33. Bazick J, Donithan M, NeuschwanderTetri BA, Kleiner D, Brunt EM, Wilson L, Doo E, Lavine J, Loomba R. Clinical Model for NASH and Advanced Fibrosis in Adult Patients With Diabetes and NAFLD: Guidelines for Referral in NAFLD. Diabetes Care 2015; 38(7): 134755.

34. Luyckx FH, Scheen AJ. Non alcoholic steatohepatitis and insulin resistance: interface between gastroenterologists and endocrinologists. Acta Clinica Belgica 2003; 58: 106-16.

35. Marchesini G, Elisabetta B, Gabriele F, Fernanda C, Marco L,Rita $M$ et al. Nonalcoholic fatty liver, steatohepatitis, and the metabolic syndrome. Hepatology 2003; 37: 917-23

36. Hsien-Liang H, Wen-Yuan L, LongTeng L, Hsih-Hsi W, Wei-Jei L. Metabolic syndrome is related to non-alcoholic steato- hepatitis in severely obese subjects. Obesity Surgery 2007; 17(11): 1457-63.

37. Gupte A, Deepak A, Subjash A, Rajiv B, Pramod K, Snehansu P, Nikhil P, Aruna M, Anjal A, Hafeez U. Non-alcoholic steatohepatitis in type 2 diabetes mellitus. Journal of Gastroenterology and Hepatology 2004; 19(8): 854-8.

38. Kenneth C. Nonalcoholic fatty liver disease in type 2 diabetes mellitus. Current Opinion in Endocrinology, Diabetes \& Obesity 2009; 16(2): 141-9.

39. Prashanth M, Ganesh HK, Vimal MV, John M, Bandga T, Shashank R J, Shah SR, Rathi PM, Josh AS, Hemangini T, Menon PM, Shah SH, Prevalence of Nonalcoholic Fatty Liver Disease in Patients with Type 2 Diabetes Mellitus. JAPI 2009; 57: 209.

40. Younossi ZM. Nonalcoholic fatty liver disease in patients with type 2 diabetes. Clin Gastroenterol Hepatol 2004; 2: 262-5.

41. Park SK. Clinical availability of nonalcoholic fatty liver disease as an early predictor of type 2 diabetes mellitus in Korean men: 5-year prospective cohort study. Hepatology 2013; 57: 1378-83.

42. Jason MH, Alex H, Geoffrey C, James G, Adamandia Kriketos, Jacob George. Beyond insulin resistance in NASH: TNF$\alpha$ or adiponectin? Hepatology 2009: 40(1): 46-54.

43. Ong JP, Elariny H, Collantes R, Younoszai A, Chandhoke V, Reines HD, Goodman Z, Younossi ZM. Predictors of non alcoholic steatohepatitis and advanced fibrosis in morbidly obese patients. Obes Surg 2005; 15: 310-5.

44. Kotronen A, Yki-Jarvinen, H. Fatty liver: a novel component of the metabolic syndrome. Arterioscler Thromb. Vasc. Biol 2008; 28: 27-38.

45. Haris EH. Elevated Liver Function Tests in Type 2 Diabetes. Clinical Diabetes 2005; 23(3): 115-9.

46. Siddharth V, Donald J, John H, Smruti RM. Predictive value of ALT levels for non-alcoholic steatohepatitis (NASH) and advanced fibrosis in non-alcoholic fatty liver disease (NAFLD). Liver International 2013; 33(9): 1398-405.

47. Kocabay GL, Telci A, Tutuncu Y, Tiryaki B, Ozel S, Cevikbas U, Okten A, Satman I. Alkaline phosphatase: can it be considered as an indicator of liver fibrosis in nonalcoholic steatohepatitis with type 2 diabetes. Bratisl Lek Listy 2011; 112(11): 626-9.

48. Sajadi

SM, Khoramdelazad $\mathrm{H}$, Hassanshahi G, Rafatpanah H, Hosseini J, Mahmoodi M, Arababadi MK, Derakhshan R, Hasheminasabzavareh R, Hosseini-Zijoud SM, Ahmadi Z. Plasma levels of CXCL1 (GRO-alpha) and CXCL10 (IP-10) are elevated in type 2 
diabetic patients: evidence for the involvement of inflammation and angiogenesis/angiostasis in this disease state. Clin Lab 2013; 59(1-2): 133-7.

49. Jingfang L, Bingyin Sh, Shuixiang $H$, Xiaoli Y, Mark DP, Zhenjun Z. Changes to tear cytokines of type 2 diabetic patients with or without retinopathy.Molecular vision 2010; 16:2931-8.

50. Xu H, Nakayama K, Ogawa S, Sugiura A, Kato T, Sato T, Sato H, Ito S. Elevated plasma concentration of IP-10 in patients with type 2 diabetes mellitus. Nihon Jinzo Gakkai shi 2005; 47(5): 524-30. 